\section{Staff and patient perspectives on unmet need and therapeutic alliance in community mental health services}

\author{
ULRICH M. JUNGHAN, MORVEN LEESE, STEFAN PRIEBE and MIKE SLADE
}

\author{
Background Therapeutic alliance \\ between clinicians and their patients is \\ important in community mental \\ healthcare. It is unclear whether providing \\ effective interventions influences \\ therapeutic alliance.
}

\begin{abstract}
Aims To assess the impact of meeting previously unmet mental health needs on the therapeutic alliance between patients and clinicians.

Method Secondary analysis of data from a longitudinal study assessing 101 patients and paired staff.
\end{abstract}

Results Patient-rated unmet need was negatively associated with patient-rated and staff-rated therapeutic alliance. Staffrated unmet need was positively associated with patient-rated therapeutic alliance only. Reducing patient-rated unmet need increased patient-rated but not staffrated therapeutic alliance, even when controlling for other variables. Reducing staff-rated unmet need increased staffrated but not patient-rated therapeutic alliance, but the effect became insignificant when controlling for other variables.

Conclusions Patient-rated therapeutic alliance will be maximised by focusing assessment and interventions on patientrated rather than staff-rated unmet need.

Declaration of interest None.

\section{Aims and hypotheses}

Therapeutic alliance between patients and clinical staff is a central component of mental healthcare (Priebe et al, 2005), and better therapeutic alliance has been found to be associated with improved outcome (Neale \& Rosenheck, 1995; Martin et al, 2000; Couture et al, 2006). Research into determinants of therapeutic alliance has mainly focused on psychotherapy. The preliminary evidence about the impact of community mental healthcare on subjective perception of therapeutic alliance suggests that the patient's perception of therapeutic alliance is related to feeling that their subjective needs are being met (Calsyn et al, 2006). A standardised assessment of mental health needs therefore offers one possibility to explore the complex patient-clinician interactions involved in therapeutic alliance (Slade et al, 2006). Modelling the longitudinal relationship between meeting previously unmet mental health needs and changes in therapeutic alliance may generate clinically relevant insights into how to improve the alliance.

\section{METHOD}

The aim of this study was to assess the cross-sectional and longitudinal impact of unmet mental health needs on therapeutic alliance, from the perspective of both patients and clinical staff. Two hypotheses were tested, each separately in relation to staff and patient perceptions of unmet need and therapeutic alliance. Hypothesis 1 (crosssectional association) was that better therapeutic alliance would be associated with fewer unmet mental health needs. Hypothesis 2 (longitudinal association) was that a reduction of unmet needs would precede an increase in therapeutic alliance.

\section{Design}

The study involved secondary analysis of data from a randomised controlled trial
(ISRCTN16971059) of routine outcome assessment and feedback to patients and staff (Slade et al, 2006).

\section{Sample and setting}

The setting was eight community mental health teams (CMHTs) in Croydon, South London. A representative random sample of 160 patients from the 3500 using adult mental health services were stratified by CMHT, diagnosis, ethnicity, age and gender. One staff member who was working most closely with each patient was then identified $(n=74$, from all main professional groups, including 43 psychiatric nurses and 14 social workers). Data were collected between 2001 and 2003, and for the current study come from the intervention group only $(n=101)$.

\section{Measures}

Unmet needs were assessed using the Camberwell Assessment of Need Short Appraisal Schedule (CANSAS; Slade et al, 1999) which assesses needs in 22 health and social domains and has separate staff (CANSAS-S) and patient (CANSAS-P) versions. Each domain is rated as either an unmet need (current serious problem, regardless of any help given), met need (no/moderate problem because of help given), no need, or not known. The unmet need score is the total number of unmet needs (range 0-22, with a high score being worse).

Therapeutic alliance was assessed using the Helping Alliance Scale (HAS), which consists of five items in the staff version (HAS-S) and six items in the patient version (HAS-P; Priebe \& Gruyters, 1993); higher scores indicate a better therapeutic alliance. The items cover basic aspects of interpersonal relationships between patients and staff as well as aspects of their judgement as to the degree of common understanding and the capability to provide or receive the necessary help respectively.

The Threshold Assessment Grid (TAG; Slade et al, 2000) is a seven-item staff-rated measure of severity of mental health problems (range 0-24, with a low score being better).

\section{Procedures}

Patients and staff were interviewed at baseline by a researcher (including round 1 data). Patients and staff were then sent postal questionnaires each month for 
5 months (rounds 2-6). The patient questionnaire included HAS-P and CANSAS-P (primary outcome), and the staff questionnaire included HAS-S and CANSAS-S. Overall postal response rates were $79 \%$ for patients and $67 \%$ for staff. Feedback on the data was sent (identically to staff and patients) after round 3 and round 6 . Follow-up assessments were completed by the researcher 1 month after the second feedback (round 7) for 93 patients in the intervention group and 92 staff. The intervention was shown to reduce admissions but not to improve CANSAS-P unmet needs (Slade et al, 2006).

\section{Analysis}

Both hypotheses were tested using multilevel random regression models (RabeHesketh \& Skrondal, 2005). These include a random effect for each individual to control for the correlation structure due to non-independence of repeated assessments. The resulting model gives a between-individuals effect (to investigate cross-sectional association) and a within-individual effect (to investigate longitudinal association).

Models were fitted with either staffrated or patient-rated therapeutic alliance as the dependent variable. The models were developed in two stages. In stage 1, the independent variables were baseline level and rating for each round of patient-rated and staff-rated unmet needs. In stage 2, the independent variables were mean patient-rated and staff-rated unmet needs over all assessments, 1-month change in patient-rated or staff-rated unmet needs (e.g. +1 meaning one more unmet need than in the previous month) and months since baseline (to investigate time trends). As a sensitivity analysis, the same independent variables as in stage 2 were used with the addition of the other unmet need change score (e.g. patient-rated unmet need change for the staff-rated therapeutic alliance model), age, gender, ethnicity, educational level, psychosis $v$. other diagnosis, TAG score and CMHT.

The effect of missing data was explored by fitting logistic regressions to a 'missing' variable, comparing missing measures on unmet needs over all assessment waves with the non-missing measurements. The robustness of models was investigated by visual inspections of the distribution of random effects. Robust estimates of the standard errors of the regression coefficients were used to estimate $P$ values and confidence intervals. All analyses were undertaken using Stata version 9.0 for Windows.

\section{RESULTS}

The relationship between therapeutic alliance and unmet mental health needs was assessed in 101 patients and paired clinical staff. Characteristics of the sample are shown in Table 1.

\section{Cross-sectional association}

Table 2 shows the patient-rated and the staff-rated therapeutic alliance models.

Low patient-rated unmet need was associated with higher staff-rated and patient-rated therapeutic alliance. In

Table I Clinical and socio-demographic characteristics of patients $(n=I 0 I)$

\begin{tabular}{|c|c|}
\hline Characteristic & \\
\hline Age, years: mean (s.d.) & $4 I .8(I I .4)$ \\
\hline Male gender, $n(\%)$ & $48(48)$ \\
\hline \multicolumn{2}{|l|}{ Ethnicity, n (\%) } \\
\hline White & $77(76)$ \\
\hline Black African-Caribbean & $16(16)$ \\
\hline Indian & $4(4)$ \\
\hline Other & $4(4)$ \\
\hline \multicolumn{2}{|l|}{ Highest educational level, $n$ (\%) } \\
\hline No formal qualifications & $38(38)$ \\
\hline GCSE or GCE' & $28(28)$ \\
\hline A-level ${ }^{2}$ & $10(10)$ \\
\hline Higher diploma or degree & II (II) \\
\hline Not known & $13(13)$ \\
\hline \multicolumn{2}{|l|}{ Primary clinical diagnosis, $n$ (\%) } \\
\hline Schizophrenia & $40(40)$ \\
\hline Bipolar affective disorder & $8(8)$ \\
\hline Other psychoses & $12(12)$ \\
\hline Affective disorder & $27(27)$ \\
\hline Personality disorder & $7(7)$ \\
\hline Other & $7(7)$ \\
\hline \multicolumn{2}{|l|}{ Contact with mental health services } \\
\hline Years since first contact: mean (s.d.) & $14.2(12.6)$ \\
\hline Years in this episode of care & $4.3(4.7)$ \\
\hline Baseline TAG score: mean (s.d.) & $5.44(3.58)$ \\
\hline \multicolumn{2}{|l|}{ CANSAS unmet need: mean (s.d.) } \\
\hline \multicolumn{2}{|l|}{ Staff-rated } \\
\hline Baseline $(n=|0|)$ & $3.24(3.31)$ \\
\hline All assessments $(n=52 \mathrm{I})$ & $2.70(3.16)$ \\
\hline \multicolumn{2}{|l|}{ Patient-rated } \\
\hline Baseline $(n=|0|)$ & $4.36(3.36)$ \\
\hline All assessments $(n=580)$ & $3.91(3.63)$ \\
\hline \multicolumn{2}{|l|}{ HAS therapeutic alliance: mean (s.d.) } \\
\hline \multicolumn{2}{|l|}{ Staff-rated } \\
\hline Baseline $(n=|0|)$ & $7.45(1.59)$ \\
\hline All assessments $(n=483)$ & $7.50(1.49)$ \\
\hline \multicolumn{2}{|l|}{ Patient-rated } \\
\hline Baseline $(n=|0|)$ & $8.19(1.79)$ \\
\hline All assessments $(n=515)$ & $7.11(2.06)$ \\
\hline
\end{tabular}

CANSAS, Camberwell Assessment of Need Short Appraisal Schedule; HAS, Helping Alliance Scale; TAG, Threshold Assessment Grid.

I. Normally taken at age 16.

2. Normally taken at age 18.
2. 
Table 2 Mixed-effects regression models of the cross-sectional impact of unmet needs on therapeutic alliance

\begin{tabular}{|c|c|c|c|c|}
\hline & \multicolumn{2}{|c|}{ Staff-rated therapeutic alliance' } & \multicolumn{2}{|c|}{ Patient-rated therapeutic alliance ${ }^{2}$} \\
\hline & B $(95 \% \mathrm{Cl})$ & $P$ & $B(95 \% \mathrm{Cl})$ & $P$ \\
\hline Baseline patient-rated unmet need & $-0.01(-0.43$ to 0.40$)$ & 0.95 & 0.17 ( -0.53 to 0.87$)$ & 0.48 \\
\hline Baseline staff-rated unmet need & $-0.40(-0.84$ to 0.03$)$ & 0.07 & $-0.45(-1.10$ to 0.19$)$ & 0.17 \\
\hline Patient-rated unmet need in each round & $-0.31(-0.54$ to -0.08$)$ & $<0.01$ & $-0.94(-1.43$ to -0.45$)$ & $<0.01$ \\
\hline Staff-rated unmet need in each round & $-0.09(-0.39$ to 0.20$)$ & 0.53 & $0.6 I$ (0.13 to I.I) & 0.01 \\
\hline Individual variation, $\sigma_{\mathrm{u}}$ & 5.77 & & 8.29 & \\
\hline Random variation, $\sigma_{e}$ & 4.03 & & 8.43 & \\
\hline Variation due to individual difference, $\rho$ & 0.67 & & 0.49 & \\
\hline Within-individual variation, $R^{2}$ & 0.02 & & 0.04 & \\
\hline Between-individual variation, $R^{2}$ & 0.14 & & 0.08 & \\
\hline Overall variation, $R^{2}$ & 0.12 & & 0.08 & \\
\hline
\end{tabular}

I. 101 participants and 304 observations.

2. 100 participants and $\mathbf{3 7 2}$ observations.

addition, low staff-rated unmet need was associated with lower patient-rated therapeutic alliance.

\section{Longitudinal association}

Table 3 shows models of the longitudinal impact of a change in unmet needs on therapeutic alliance.

Higher mean levels of unmet needs were associated with lower therapeutic alliance in both models, consistent with the cross-sectional association already shown. In addition, a decrease in the number of patient-rated unmet needs was associated with higher patient-rated therapeutic alliance in the month following this change, and a decrease in the number of staff-rated unmet needs was associated with higher staff-rated therapeutic alliance in the following month. There was a significant improvement in both staff-rated and patient-rated therapeutic alliance over time. In all models the proportion of unexplained variance $(\rho)$ attributable to individual differences was high (ranging from 49 to $78 \%)$.

\section{Sensitivity analyses}

In a sensitivity analysis, each model was estimated using both unmet need change scores and including clinical and demographic variables $(n=77$ for staff model, $n=71$ for patient model). The resulting models were similar. Change in staffrated unmet need did not have an impact on patient-rated therapeutic alliance
$(B=-0.12, \quad P=0.96)$ and change in patient-rated unmet need did not have an impact on staff-rated therapeutic alliance $(B=-0.02, \quad P=0.73)$. Improvement in patient-rated unmet need remained associated with better patient-rated therapeutic alliance $(B=-40, P=0.03)$ but the previous association between staff-rated unmet need change and staff-rated therapeutic alliance became insignificant $(B=-0.23, P=0.06)$. The only clinical or demographic variable with a significant effect was CMHT which had an effect on staff-rated therapeutic alliance. Two of the eight CMHTs had a significant tendency towards more negative ratings for therapeutic alliance. The impact of CMHT was investigated using a threelevel random mixed model, with patients nested in CMHTs and repeated measures

Table 3 Mixed-effects regression models of the longitudinal effects of changing unmet needs on therapeutic alliance

\begin{tabular}{|c|c|c|c|c|}
\hline & \multicolumn{2}{|c|}{ Staff-rated therapeutic alliance ${ }^{\prime}$} & \multicolumn{2}{|c|}{ Patient-rated therapeutic alliance ${ }^{2}$} \\
\hline & B $(95 \% \mathrm{Cl})$ & $P$ & $B(95 \% \mathrm{Cl})$ & $P$ \\
\hline Mean level of unmet needs over all assessments ${ }^{3}$ & $-0.85(-1.38$ to -0.32$)$ & $<0.01$ & $-1.35(-2.06$ to -0.65$)$ & $<0.01$ \\
\hline Change in unmet needs over preceding month $\mathrm{h}^{3,4}$ & $-0.16(-0.90$ to -0.11$)$ & 0.05 & $-0.51(-0.90$ to -0.11$)$ & 0.01 \\
\hline Linear trend & $0.30(0.07$ to 0.53$)$ & $<0.01$ & $0.66(0.2 I$ to I.II) & $<0.01$ \\
\hline Individual variation, $\sigma_{u}$ & 6.34 & & 8.56 & \\
\hline Random variation, $\sigma_{\mathrm{e}}$ & 3.27 & & 6.83 & \\
\hline Variation due to individual difference, $\rho$ & 0.78 & & 0.61 & \\
\hline Within-individual variation, $R^{2}$ & 0.038 & & 0.075 & \\
\hline Between-individual, $R^{2}$ & 0.108 & & 0.148 & \\
\hline Overall variation, $R^{2}$ & 0.141 & & 0.143 & \\
\hline
\end{tabular}

I. 88 participants and 304 observations.

2. 88 participants and 372 observations.

3. Patient-rated unmet need for patient-rated therapeutic alliance model, staff-rated unmet need for staff-rated therapeutic alliance model.

4. Change in unmet need: $+I$ means a rise in unmet needs from the previous month by $I$. 
nested in individual patients. The resulting model (not shown) was similar to that in Table 2. Fitting a logistic regression model to a 'missing' variable did not show any systematic differences between the characteristics of those with missing assessments and those included in our analyses, except that one out of eight CMHTs had fewer missing assessments.

\section{DISCUSSION}

This study investigated the relationship between patient and staff perceptions of unmet need and therapeutic alliance. Lower patient-rated unmet need was cross-sectionally associated with higher staff-rated and patient-rated therapeutic alliance, whereas lower staff-rated unmet need was only associated with lower (not higher) patientrated therapeutic alliance. Longitudinally, a decrease in patient-rated unmet need was associated with higher patient-rated therapeutic alliance, a relationship which remained when controlling for other needs, clinical and demographic variables. A decrease in staff-rated unmet need was also associated with higher staff-rated therapeutic alliance, but the relationship became insignificant when other variables were controlled for.

\section{Therapeutic alliance in community mental health settings}

The relationship between therapeutic alliance and a range of mental health outcomes has been extensively researched. Improved therapeutic alliance has been repeatedly associated with improved outcome. The available evidence has two limitations when applied in community mental health settings, which the current study addresses.

First, most studies have been in psychotherapy settings (e.g. Martin et al, 2000). Routine community mental healthcare differs from psychotherapy in several ways, including an emphasis on meeting both health and social needs, multi-professional and multi-staff input, and providing practical help and social support. Studies investigating therapeutic alliance between a patient and a psychotherapist may not, therefore, generalise to community mental health settings (Priebe \& McCabe, 2006).

Second, existing studies have not used a repeated-measures design, which limits the ability to understand the relationship between different process and outcome domains (e.g. Neale \& Rosenheck, 1995).
There are complex determinants of therapeutic alliance in community mental healthcare (e.g. Priebe \& McCabe, 2006). For example, qualitative studies identify influences, such as being heard, having meaningful choices between treatment options and being actively helped, as all associated with better therapeutic alliance (McCabe et al, 2002; Ware et al, 2004). If the investigation of therapeutic alliance in community mental health services is to progress, then empirically-based conceptual models are needed.

\section{Preliminary model of unmet need and therapeutic alliance}

Bordin (1979) proposed that therapeutic alliance has three components: goals, bonds and tasks. High therapeutic alliance is present where there is agreement on the goals of therapy, strong patient-therapist bonds (e.g. trust, respect) and positive views about the methods of working (e.g. therapist's skills, patients' perception of the therapist's ability to help them). Unmet need provides candidate proxy measures for two elements of Bordin's tripartite framework: identifying an unmet need is a proxy for therapeutic goals and meeting previously unmet needs is a proxy measure for task effectiveness. Therefore, the relationship between unmet need and therapeutic alliance is worth exploring.

Empirical evidence indicates the need to consider staff and patient assessments separately. Views about the level of therapeutic alliance differ between staff and patient (Bale et al, 2006). Similarly, disagreement exists between individual staff-patient pairs in relation to the number and nature of unmet needs (e.g. Gibbons et al, 2005; Cleary et al, 2006; Fleury et al, 2006). Therefore, both perspectives need to be investigated when exploring the relationship between therapeutic alliance and unmet needs.

The current study provides the first evidence of a cross-sectional and longitudinal association between unmet need and therapeutic alliance. This is the first empirical study to identify an approach to improving therapeutic alliance in routine community care. Meeting unmet needs (especially patient-rated needs) was followed by improvements in therapeutic alliance. The results of this study are not compatible with an explanation that the relationship between unmet need and therapeutic alliance arises from an unknown mediator, such as treatment adherence. First, the relationship is stable across the different models. Second, the reverse model (testing whether change in therapeutic alliance predicts unmet need) did not fit the data.

Is there evidence that meeting needs improves outcome? Reducing unmet needs in people with severe mental illness has been shown to be associated with improved quality of life, and this relationship is strongest for patient-rated (rather than staffrated) unmet need (Lasalvia et al, 2005; Slade et al, 2005). These findings have led to the suggestion that need may be 'the mediating link between subjective quality of life and all its influences (rather than just psychiatric influences)' (Slade et al, 2004: p. 188). Our results suggest a more refined model, in which the relationship between patient-rated unmet need and quality of life is mediated by improved therapeutic alliance. This model is consistent with studies in other areas of medicine. For example, Howard et al (2006) showed that the relationship between interpersonal problems and depression in people with multiple sclerosis was mediated by therapeutic alliance.

\section{Strengths of the study}

A strength of the present study is its longitudinal repeated-measures design which allows investigation of the temporal relationship between therapeutic alliance and unmet needs over time, which is not possible with cross-sectional or pre-post assessments (Pearl, 2000). Our results showed that there is a relationship between unmet need and therapeutic alliance, and that change in patient-rated unmet need precedes change in therapeutic alliance. The nature of this relationship could be investigated in a randomised controlled trial (RCT) with an intervention to meet previously unmet needs. Supplementing the trial with repeated measures of therapeutic alliance before and after the intervention - akin to an interrupted time series (Gilbody \& Whitty, 2002) - would allow investigation of whether the relationship between unmet need and therapeutic alliance is causal (Bollen, 1989), and strengthening evidence on approaches to improving the alliance.

A second strength of the study is in representativeness. The recruitment strategy for the study ensured participants were representative of those using local CMHTs and the sample setting was chosen to be 
demographically typical for England (Slade et al, 2006). Similarly, the majority of the data were collected by post rather than by interview. Results are therefore likely to be of general relevance.

\section{Limitations of the study}

Three limitations can be identified. First, data came from an RCT which was investigating the use of monthly assessments by staff and patients plus 3-monthly feedback (including unmet need and therapeutic alliance) to staff and patients. It is possible that the intervention influenced the ratings of unmet need and therapeutic alliance. However, in the RCT there were no significant differences between the intervention and control groups in therapeutic alliance or unmet needs at follow-up. Furthermore, a cross-sectional inverse relationship between unmet needs and therapeutic alliance was also present when we analysed the baseline and follow-up assessments of the control group $(n=59 ; B=-0.92, P<0.01$ for patients; $B=-0.71, P<0.01$ for staff). There was, therefore, no evidence that the relationship between unmet need and therapeutic alliance was influenced by the intervention.

Second, we found a systematic difference between therapeutic alliance in the CMHTs and, although it may reflect different case-load compositions, it is a potential source of bias. However, including the CMHT as a variable in the model did not fundamentally affect the pattern of relationship between unmet needs and therapeutic alliance.

Third, unmet needs only explain a moderate portion of the variance in therapeutic alliance. Meeting 1 patient-rated unmet need of the 22 assessed was followed by a change of half a point (scale 1-6) in patient-rated therapeutic alliance. The high levels of unexplained variance attributable to individual differences in the random regression models indicate that there are other important individual-level determinants of staff-rated and patient-rated therapeutic alliance that were not considered in this study. Therefore, future research on therapeutic alliance in community mental healthcare should also explore other determinants, such as symptomatology, level of agreement on need, and characteristics of staff and CMHT.

\section{Implications}

This study found that meeting patient-rated unmet needs leads to better therapeutic

ULRICH M. JUNGHAN, MD, University Hospital of Psychiatry, Unit of Community Psychiatry, Bern, Switzerland; MORVEN LEESE, PhD, Institute of Psychiatry, King's College London; STEFAN PRIEBE, PhD, Department of Psychiatry, Queen Mary, University of London and Newham Centre for Mental Health, London; MIKE SLADE, $\mathrm{PhD}$, Institute of Psychiatry, King's College London, UK

Correspondence: Dr Ulrich Junghan, University Hospital of Psychiatry, Unit of Community Psychiatry, Laupenstrasse 49,CH-3010 Bern, Switzerland. Email: junghan@spk.unibe.ch

(First received I5 March 2007, final revision 9 July 2007, accepted 23 August 2007)

alliance. A reduction of one patient-rated unmet need resulted in an improvement of patient-rated therapeutic alliance by half a point (scale range $0-10$ ), and hence meeting five unmet needs (range $0-22$ ) will lead to a clinically significant improvement (HAS-P s.d. $=2.07$, assuming reliability of 0.8 ; Jacobsen \& Truax, 1991). Therefore, if one goal of care is to maximise therapeutic alliance and hence engagement, then treatment planning should be at least partly driven by patient rather than staff perspectives on need.

\section{REFERENCES}

Bale, R., Catty, J., Watt, H., et al (2006) Measures of the therapeutic relationship in severe psychotic illness: a comparison of two scales. International Journal of Social Psychiatry, 52, 256-266.

Bollen, K. (1989) Structural Equations with Latent Variables. Wiley

Bordin, E. (1979) The generalizability of the psychoanalytic concept of the working alliance. Psychotherapy: Theory, Research and Practice, 16, 252-260.

Calsyn, R. J., Klinkenberg, W. D., Morse, G. A., et al (2006) Predictors of the working alliance in community treatment. Community Mental Health Journal, 42 , $161-175$.

Cleary, M., Friedman, A., Hunt, G., et al (2006)

Patient and carer perceptions of need and associations with care-giving burden in an integrated mental health service. Social Psychiatry and Psychiatric Epidemiology, 4I, 208-214.

Couture, M. S., Roberts, D. L., Penn, D. L., et a (2006) Do baseline characteristics predict the therapeutic alliance in the treatment of schizophrenia. Journal of Nervous and Mental Disease, 94, 10-14.

Fleury, M., Grenier, G. \& Lesage, A. (2006) Agreement between staff and service users concerning the clientele's mental health needs: a Quebec study. Canadian Journal of Psychiatry, 5I, 28I-286.

Gibbons, C., Bédard, M. \& Mack, G. (2005) Comparison of client and mental health worker assessment of needs and unmet needs. Journal of Behavioral Health Services and Research, 32, 95-104.

Gilbody, S. \& Whitty, P. (2002) Improving the delivery and organisation of mental health services: beyond the conventional randomised controlled trial. British Journal of Psychiatry, 180, 13-18.

Howard, I., Turner, R., Olkin, R., et al (2006) Therapeutic alliance mediates the relationship between interpersonal problems and depression outcome in a cohort of multiple sclerosis patients. Journal of Clinical Psychology, 62, 1197-1204.
Jacobsen, N. \& Truax, P. (1991) Clinical significance: a statistical approach to defining meaningful change in psychotherapy research. Journal of Consulting and Clinical Psychology, 59, 12-19.

Lasalvia, A., Bonetto, C., Malchiodi, F., et al (2005) Listening to patients' needs to improve their subjective quality of life. Psychological Medicine, 35, I-II.

Martin, D., Garske, J. \& Davis, M. (2000) Relation of the therapeutic alliance with outcome and other variables: a meta-analytic review. Journal of Consulting and Clinical Psychology, 68, 438-450.

McCabe, R., Heath, C., Burns, T., et al (2002) Engagement of patients with psychosis in the consultation: conversation analytic study. BMJ, $\mathbf{3 2 5}$ ||48-||5|.

Neale, M. \& Rosenheck, R. (1995) Therapeutic alliance and outcome in a VA intensive case management program. Psychiatric Services, 46, 719-72I.

Pearl, J. (2000) Causality: Models, Reasoning and Inference. Cambridge University Press.

Priebe, S. \& McCabe, R. (2006) The therapeutic relationship in psychiatric settings. Acta Psychiatrica Scandinavica Supplementum, 429, 69-72.

Priebe, S., Watts, J., Chase, M., et al (2005) Processes of disengagement and engagement in assertive outreach patients: qualitative study. British Journal of Psychiatry, 187, 438-443.

Priebe, S. \& Gruyters, T. (1993) The role of the helping alliance in community care - a prospective study. Journal of Nervous and Mental Disease, 181, 552-557.

Rabe-Hesketh, S. \& Skrondal, A. (2005) A Multilevel and Longitudinal Modeling using Stata. Stata Press.

Slade, M., Loftus, L., Phelan, M., et al (1999) The Camberwell Assessment of Need. Gaskell.

Slade, M., Powell, R., Rosen, A., et al (2000)

Threshold Assessment Grid (TAG): the development of a valid and brief scale to assess the severity of mental illness. Social Psychiatry and Psychiatric Epidemiology, 35. 78-85.

Slade, M., Leese, M., Ruggeri, M., et al (2004) Does meeting needs improve quality of life? Psychotherapy and Psychosomatics, 73, 183-189.

Slade, M., Leese, M., Cahill, S., et al (2005) Patientrated mental health needs and quality of life improvement. British Journal of Psychiatry, 187, 256-26I.

Slade, M., McCrone, P., Kuipers, E., et al (2006) Use of standardised outcome measures in adult mental health services: randomised controlled trial. British Journal of Psychiatry, 189, 330-336.

Ware, N., Tugenberg, T. \& Dickey, B. (2004)

Practitioner relationships and quality of care in lowincome persons with serious mental illness. Psychiatric Services, 55, 555-559. 